\title{
Blunt Aortic / Inferior Vena Cava Injury: Are We Consistently Providing the Same Level of Care?
}

\author{
Monica Leon ${ }^{1}$, Luis O. Chavez ${ }^{2}$, Alda Chavez ${ }^{3}$, Salim Surani ${ }^{4,5,6}$ \\ 1. General Surgery, ABC Medical Center, Mexico City, MEX 2. Internal Medicine, Texas Tech University Health Sciences \\ Center, El Paso, USA 3. Faculty of Medicine and Psychology, Autonomous University of Baja California, Tijuana, MEX \\ 4. Internal Medicine, Texas A\&M Health Science Center, Bryan, USA 5. Internal Medicine, Corpus Christi Medical \\ Center, Corpus Christi, USA 6. Internal Medicine, University of North Texas, Dallas, USA
}

Corresponding author: Salim Surani, srsurani@hotmail.com

\begin{abstract}
Major vascular traumatic injuries have a higher pre-hospital and in-hospital mortality rate. The different mechanisms of injury and anatomy of the aorta and inferior vena cava (IVC) make the management a constant challenge to surgeons and clinicians. Blunt traumatic aortic injury (BTAI) can occur at the thoracic or abdominal level, each of which possesses different considerations. Blunt traumatic inferior vena cava injury (BTIVCI) also has important diagnostic challenges since the lesion may not be as evident in the IVC as compared to the aorta, possibly due to lower caval pressures or the ability to self-tamponade from adjacent structures. Endovascular management has significantly increased in the past years, and despite an improvement in mortality, the approach to aortic and IVC injuries is not well standardized. Diagnostic imaging helps to classify the extent of the lesions and guide towards the best therapeutic options for each case. Conservative management, in some cases, has shown to reduce mortality, and close follow-up has proven good outcomes. Future research will provide more evidence to determine the best approach to BTAI and BTIVCI for better long-term outcomes. This article aims to provide an updated review of the current literature regarding diagnosis, classification, and management of BTAI and BTIVCI.
\end{abstract}

Categories: Cardiac/Thoracic/Vascular Surgery, Emergency Medicine, Trauma

Keywords: blunt trauma, aortic injury, inferior vena cava injury, vascular injury, shock, ivc

\section{Introduction And Background}

Trauma-related injuries are a leading cause of death worldwide that have increased over the last two decades [1]. Major vascular trauma such as injuries to the aorta or the inferior vena cava (IVC) has a high rate of prehospital mortality [2]. Blunt traumatic aortic injury (BTAI) is the second most common post-traumatic cause of death following traumatic brain injury as the first cause. According to the National Trauma Databank of the American College of Surgeons, from 2002-2014 the incidence of combined injuries to the IVC and the thoracic aorta was $1.1 \%$, thoracic and abdominal aorta was $0.8 \%$ and IVC and abdominal aorta was $2.1 \%$ [1].

Received 01/16/2020 Review began 01/27/2020 Review ended 01/27/2020 Published 01/31/2020

\section{(c) Copyright 2020}

Leon et al. This is an open access article distributed under the terms of the Creative Commons Attribution License CC-BY 3.0., which permits unrestricted use, distribution, and reproduction in any medium, provided the original author and source are credited.
In the past, the standard of care of traumatic vascular injuries was open surgical repair, and the in-hospital mortality for BTAI in the first 24 hours was up to $50 \%$ of patients [3, 4]. However, in the last two decades, endovascular management for blunt trauma vascular injuries has significantly increased from $0.4 \%$ in 2002 to $13.2 \%$ in 2010 . Parallel to these findings, the overall mortality from aortic and IVC blunt trauma decreased from $48.8 \%$ in 2002 to $28.7 \%$ in 2014, with a more significant reduction in the BTAI group [1]. The use of endovascular techniques has decreased morbidity and mortality from traumatic blunt vascular trauma $[1,5]$. The aim of this article is to provide an updated literature review of the diagnosis, classification and current management of both BTAI and blunt traumatic inferior vena cava injury (BTIVCI).

\section{Review}

\section{Blunt traumatic aortic injury}

Mechanism of injury in blunt trauma to aorta includes tearing of the aorta that is pinched between the vertebral body or displaced sternum, torsion and shearing forces, rapid deceleration and increased intravascular pressure. The most common site of injury is the aortic isthmus, followed by the ascending aorta and diaphragmatic hiatus. During the initial evaluation, findings suggestive of BTAI in a chest radiograph (CXR) include mediastinal widening (more than eight centimeters) at the aortic arch, irregularity of the aortic arch, loss of definition of the aortopulmonary window, deviation of the trachea, widening of left para-tracheal stripe, among others [3].

Since CXR only provides suggestive findings and lacks abdominal visualization, computed tomography angiography (CTA) is the imaging of choice for BTAI, with a reported sensitivity of $98 \%$ and specificity of nearly 100\% [3]. CTA imaging findings are divided as suggestive and definitive signs for traumatic aortic injury. Suggestive signs are mediastinal or peri-aortic hematoma, retro-crural hematoma, and small caliber of the aorta distal to the injury. Definitive signs are aortic dissection (Figure 1), contained rupture, 


\section{Cureus}

intramural thrombus, active contrast extravasation (Figure 2), and abnormalities of aortic contour [3].

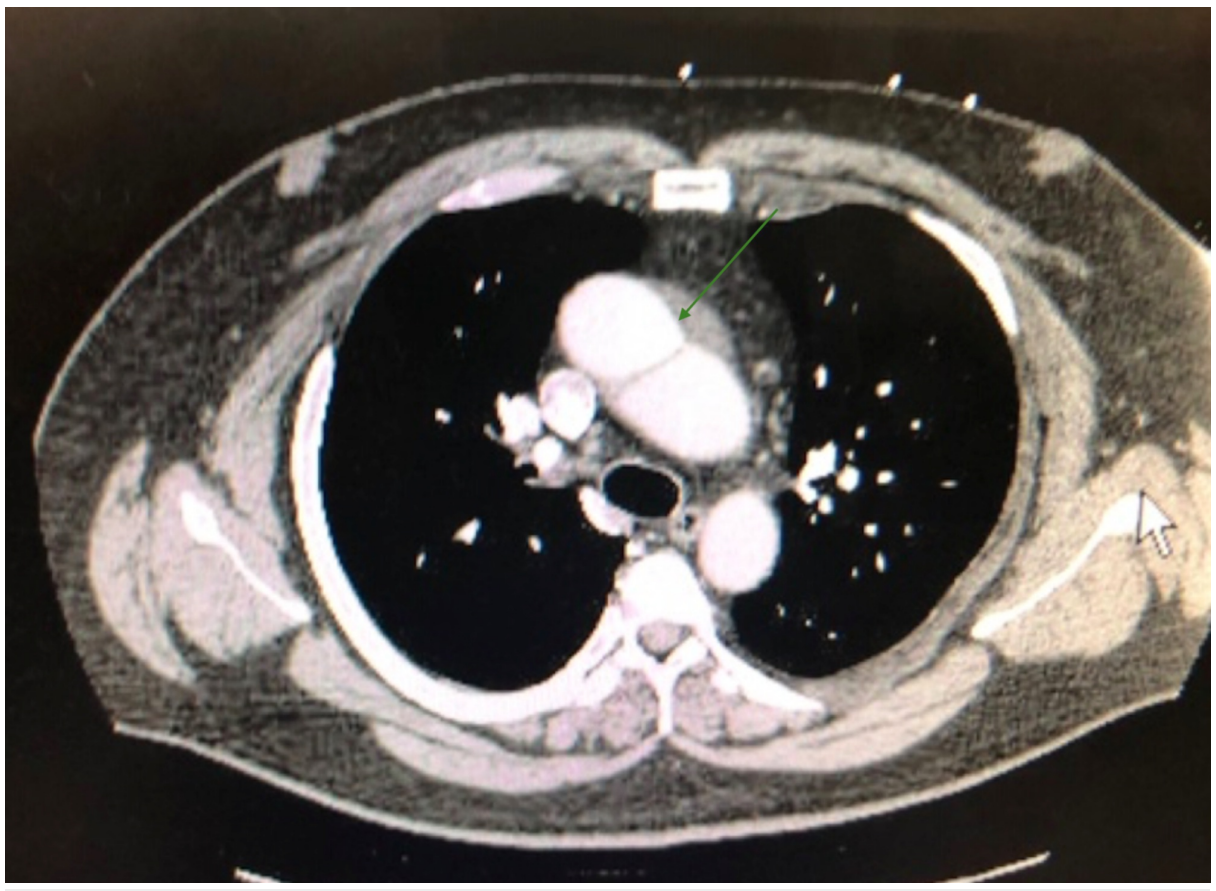

FIGURE 1: Ascending aorta dissection

CT scan of the chest with intravenous contrast showing the aortic dissection.

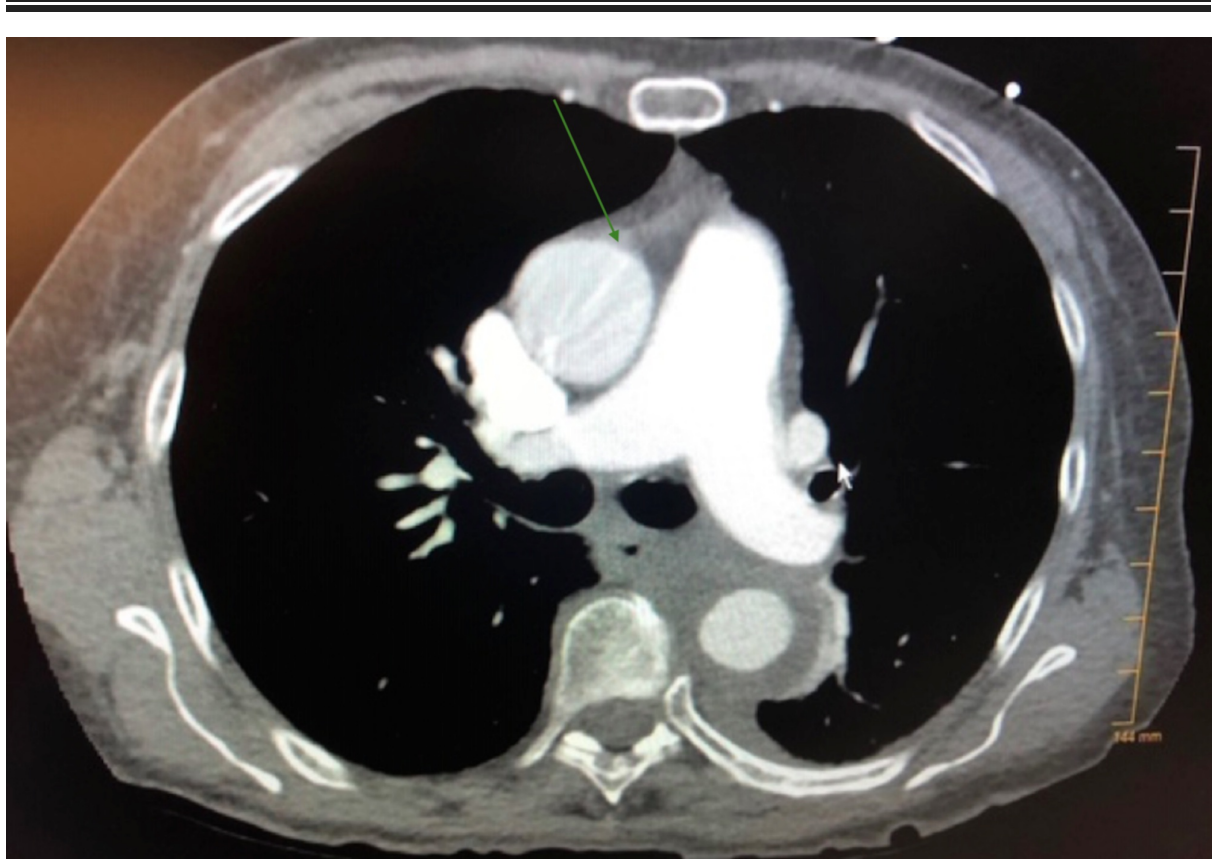

FIGURE 2: Aortic dissection with extravasation

CT scan of the chest with intravenous contrast showing aortic dissection with extravasation.

Classifications

Multiple classifications have been proposed over the years (Table 1). Parmley's classification (1958) is probably the first reported in the literature [6]. This classification is obsolete nowadays because it is pathologically based. Classifications based on CT findings are currently used. For many years, one of the most commonly used classifications was the one proposed by Azizzadeh et al [7]. A modification to this 


\section{Cureus}

classification was proposed by the Society of Vascular Surgery as intramural hematoma (Grade II) is radiographically poorly defined and can be associated with injuries of adjacent structures and may not represent a true aortic injury [8].

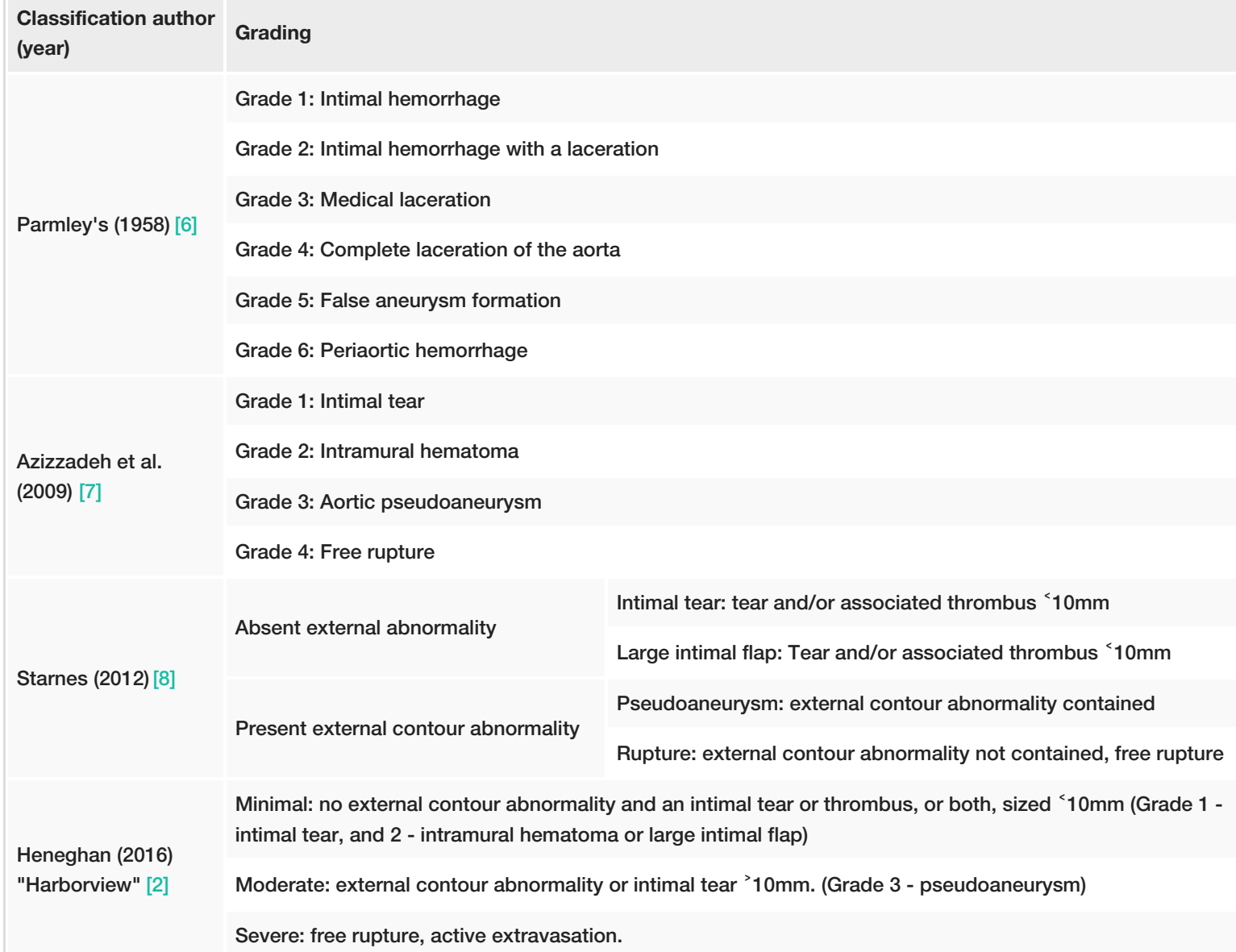

TABLE 1: Traumatic aortic injury classifications

The term "minimal aortic injury" (MAI) was coined by Malhotra et al. in 2001 [9]. It was used to describe an intimal flap of less than one centimeter with minimal or no periaortic hematoma. MAI is usually underdiagnosed by CT and diagnosed more accurately with higher-resolution techniques of CTA, intravascular ultrasound (IVUS) or angiography. The term MAI has been used in different studies with varying definitions [3]. Recently, the "Harborview" classification was proposed by Heneghan et al., which includes the term MAI and guides management based on the grade of injury [2].

\section{Thoracic Aorta}

The pre-hospital mortality of BTAI in the thoracic segment is approximately $85 \%$. In-hospital mortality is approximately $19 \%$ with open repair and $3 \%$ with endovascular repair [10]. According to the Society of Vascular Surgery guidelines from 2011, Grade 1 injuries (intimal tear) warrant expectant management with serial imaging, while Grades 2-4 should undergo repair with thoracic endovascular aortic repair (TEVAR) (Figure 3) [11]. TEVAR has successfully replaced open surgical repair by providing better outcomes, decreasing mortality and minimizing complications associated with open surgical repair (spinal cord ischemia, thoracotomy, aortic cross-clamping and the use of cardiopulmonary bypass) [10, 11]. 


\section{Cureus}

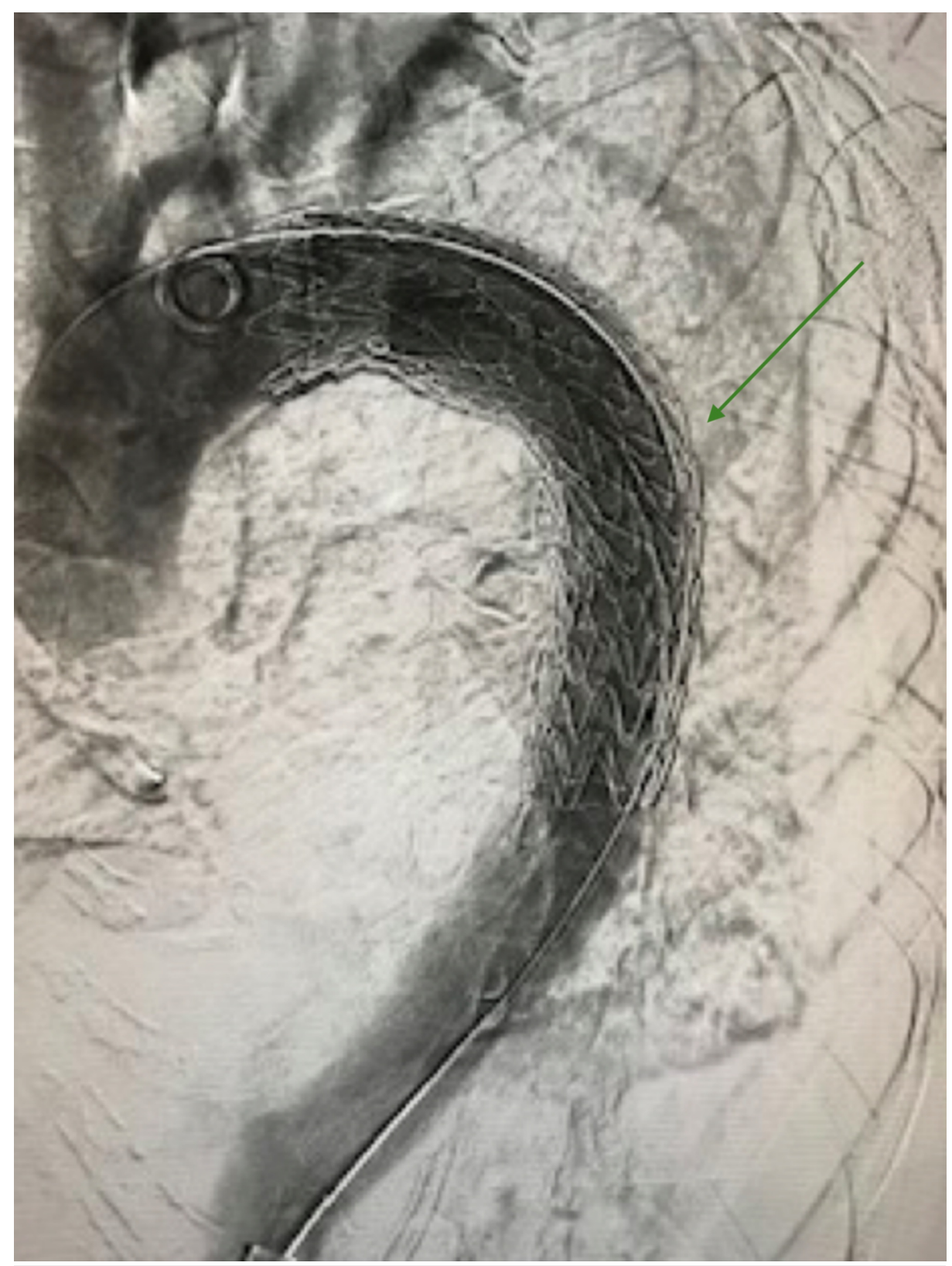

\section{FIGURE 3: Thoracic endograft}

New treatment strategies for BTAI are leaning towards a more conservative approach. Initial medical management focuses on blood pressure and heart rate control which are the determinants of aortic wall stress, dissection extension and rupture [12, 13]. Targets are usually a heart rate less than $60 \mathrm{bpm}$ and systolic blood pressure between 100-120 mmHg [13]. Beta-blockers are usually the preferred group of agents to control these parameters. For some patients, vasodilators can be required as secondary agents to control blood pressure (Table 2) [12]. 


\section{Cureus}

\begin{tabular}{|ll|}
\hline Medica therapy & \\
\hline Anti-impulse therapy (Beta-blockers) & Propanolol \\
& Metoprolol \\
& Labetalol $^{*}$ \\
Vasodilators & Esmolol $^{\star *}$ \\
& Sodium nitroprusside \\
\hline & Nicardipine \\
\hline
\end{tabular}

\section{TABLE 2: Medical therapy}

* Alpha and beta receptor antagonist, the advantage of heart rate and blood pressure control from a single agent.

${ }^{\star *}$ Viable option for patients with potential contraindication to beta-blockade due to extremely short half-life.

Delayed aortic endovascular repair (after the first 24 hours) is usually reserved for high-risk patients with associated traumatic injuries. Recently, many studies have shown a decrease in mortality in BTAI stable patients with delayed repair [14]. The Eastern Association for Surgery of Trauma recommends delayed repair with effective blood pressure control in this subset of patients [4].

Recommendations for the management of BTAI Grades 1-2 injuries include antiplatelet therapy (aspirin 81 $\mathrm{mg}$ for four to six weeks) with no surgical intervention and follow up imaging [2]. Management of Grade 3 (moderate injuries) is based on semi-elective repair during the first 24-72 hours along with optimization of blood pressure and prompt initiation of antiplatelet therapy. Emergency thoracic endovascular aneurysm repair (TEVAR) is reserved for severe (Grade 4) injuries [14].

\section{Abdominal Aorta}

Abdominal BTAI accounts for only $2.5-5 \%$ of blunt aortic trauma $[1,15,16]$. The abdominal aorta extends from the diaphragmatic hiatus to the aortic bifurcation. BTAI is more common at the level of the inferior mesenteric artery (33\%) followed by lesions at the level of the renal arteries (24\%) [17]. Mortality from blunt abdominal aorta trauma has decreased in the US from $58.3 \%$ in 2002 to $26.2 \%$ in the last two decades [1].

Although aortic injury classifications are initially based on thoracic injuries, abdominal injuries can be described with the same classification. Intimal flaps $<10 \mathrm{~mm}$ are safely managed with blood pressure control (B-blockers) and antiplatelet therapy (aspirin) [16, 18]. Large intimal flaps, not complicated with thrombosis or arterial insufficiency, are treated with medical management and follow-up CT imaging in 48-72 hours. Large intimal flaps with complications, pseudo-aneurysms, and frank rupture, require urgent endovascular or open surgical repair [16]. Trends are similar to thoracic BTAI management, with an increasing endovascular approach, as an open surgical repair is associated with higher mortality [1, 19]. The use of the endovascular approach for abdominal aortic blunt trauma repair increased from $1.9 \%$ in 2002 to $15.9 \%$ in 2014 with associated improvement in survival [1].

\section{Blunt traumatic inferior vena cava injury}

Blunt traumatic inferior vena cava injury (BTIVCI) occurs only in 1-3.2\% of trauma patients [1, 20]. The inferior vena cava (IVC) is divided into infra-renal, para-renal, supra-renal, retro-hepatic, and suprahepatic. The infra-renal segment is the most commonly injured segment in approximately $39 \%$. The segments with the highest mortality when injured are the retro-hepatic and supra-hepatic [21].

Hospital mortality rates from BTIVCI vary widely, with reports ranging from $49 \%$ to $70 \%$ [1, 22]. BTIVCI has greater mortality than IVC penetrating injury, abdominal aorta and thoracic aorta [1]. This could be explained by delayed presentation, difficult diagnosis and difficult surgical exposure for repair. Injuries closer to the heart are associated with higher mortality [23]. Hemorrhagic shock, Glasgow Coma Scale and associated injuries are independent factors related to higher mortality rates in BTIVCI [24]. 
ranging from avulsion of the atrio-caval junction to tearing or intra-parenchymal lacerations [21, 25]. Violent shearing forces contribute to larger and irregular lacerations than the ones observed with IVC penetrating injury [25]. More than half of IVC injuries spontaneously contain the site of injury with the cessation of bleeding by self-tamponade [21]. In stable patients who undergo CT scan, direct signs of IVC injury (such as contrast extravasation) are not easily identified, possibly due to the low caval pressure or tamponade from adjacent structures [22]. However, indirect signs that may help with diagnosis include pericaval hematoma, irregular caval contour, and filling defects [22, 26, 27].

Management of BTIVCI depends on the hemodynamics of the patient, the location and extent of the lesion and the associated comorbidities. Treatment modalities include definitive vascular repair, damage control procedures (ligation, packing) or conservative approach. The latter usually is restricted to patients who are stable and whose injuries have self-tamponade or contained by adjacent organs (usually the liver, suspensory ligaments, and the diaphragm) [20, 22, 28]. Endovascular therapy for BTIVCI has also up-trended through the years from $0.3 \%$ in 2000 to $4.2 \%$ in 2014 in the United States. This advance in management is of extreme importance since in-hospital mortality for BTIVCI is most commonly caused by intraoperative exsanguination $[21,29]$. BTIVICI with pseudo-aneurysm and/or free rupture has been successfully treated with an endovascular approach [30].

The natural history of IVC injuries is not well known and there are fewer reports of follow up compared to aortic injuries. However, conservative management and a combination of endovascular and open repair have been reported in the literature. Cheaito et al. reported a case of a pseudo-aneurysm at the hepatic confluence of the IVC treated conservatively with the total resolution at six months follow-up [20]. Watarida et al. reported the use of a fenestrated stent-graft for blunt traumatic injury of the juxtahepatic IVC with successful control of massive retroperitoneal hematoma with active bleeding [31].

Management with early endovascular management for hemorrhage control, followed by open repair with good results, has also been reported [29].

\section{Conclusions}

Blunt traumatic aortic and IVC injuries remain a leading cause of death worldwide. The complexity of the anatomy and mechanism of injury of these vascular lesions make it difficult to provide a standardized approach. However, the trend in management is shifting towards less aggressive approaches in the proper clinical scenarios. Endovascular management has yielded positive outcomes and correlated with significant improvement in in-hospital survival. A multi-disciplinary team is mandatory for the care of both blunt traumatic aortic and IVC injuries since some cases require hybrid management with endovascular and open surgical repair. Update in guidelines is foreseen in the upcoming years as more techniques are being developed, and more evidence is gathered. Future research will help determine in which cases conservative management provides better outcomes as compared to early interventions.

\section{Additional Information \\ Disclosures}

Conflicts of interest: In compliance with the ICMJE uniform disclosure form, all authors declare the following: Payment/services info: All authors have declared that no financial support was received from any organization for the submitted work. Financial relationships: All authors have declared that they have no financial relationships at present or within the previous three years with any organizations that might have an interest in the submitted work. Other relationships: All authors have declared that there are no other relationships or activities that could appear to have influenced the submitted work.

\section{References}

1. Branco BC, Musonza T, Long MA, et al.: Survival trends after inferior vena cava and aortic injuries in the United States. J Vasc Surg. 2018, 68:1880-1888. 10.1016/j.jvs.2018.04.033

2. Heneghan RE, Aarabi S, Quiroga E, Gunn ML, Singh N, Starnes BW: Call for a new classification system and treatment strategy in blunt aortic injury. J Vasc Surg. 2016, 64:171-176. 10.1016/j.jvs.2016.02.047

3. Nagpal P, Mullan BF, Sen I, Saboo SS, Khandelwal A: Advances in imaging and management trends of traumatic aortic injuries. Cardiovasc Intervent Radiol. 2017, 40:643-654. 10.1007/s00270-017-1572-X

4. Fox N, Schwartz D, Salazar JH, et al.: Evaluation and management of blunt traumatic aortic injury: a practice management guideline from the Eastern Association for the Surgery of Trauma. J Trauma Acute Care Surg. 2015, 78:136-146. 10.1097/TA.0000000000000470

5. Khoynezhad A, Donayre CE, Azizzadeh A, White R: RESCUE investigators: one-year results of thoracic endovascular aortic repair for blunt thoracic aortic injury (RESCUE trial). J Thorac Cardiovasc Surg. 2015, 149:155-161. 10.1016/j.jtcvs.2014.09.026

6. Parmley LF, Mattingly TW, Manion WC, Jahnke Jr EJ: Nonpenetrating traumatic injury of the aorta . Circulation. 1958, 17:1086-1101.10.1161/01.cir.17.6.1086

7. Azizzadeh A, Keyhani K, Miller CC 3rd, Coogan SM, Safi HJ, Estrera AL: Blunt traumatic aortic injury: initial experience with endovascular repair. J Vasc Surg. 2009, 49:1403-1408. 10.1016/j.jvs.2009.02.234

8. Starnes BW, Lundgren RS, Gunn M, et al.: A new classification scheme for treating blunt aortic injury . J Vasc Surg. 2012, 55:47-54. 10.1016/j.jvs.2011.07.073 
9. Malhotra AK, Fabian TC, Croce MA, Weiman DS, Gavant ML, Pate JW: Minimal aortic injury: a lesion associated with advancing diagnostic techniques. J Trauma. 2001, 51:1042-1048. 10.1097/00005373200112000-00003

10. Reuben BC, Whitten MG, Sarfati M, Kraiss LW: Increasing use of endovascular therapy in acute arterial injuries: analysis of the National Trauma Data Bank. J Vasc Surg. 2007, 46:1222-1226. 10.1016/j.jvs.2007.08.023

11. Lee WA, Matsumura JS, Mitchell RS, et al.: Endovascular repair of traumatic thoracic aortic injury: clinical practice guidelines of the Society for Vascular Surgery. J Vasc Surg. 2011, 53:187-192. 10.1016/j.jvs.2010.08.027

12. Hiratzka LF, Bakris GL, Beckman JA, et al.: 2010 ACCF/AHA/AATS/ACR/ASA/SCA/SCAI/SIR/STS/SVM guidelines for the diagnosis and management of patients with thoracic aortic disease. Circulation. 2010, 121:266-369. 10.1161/CIR.0b013e3181d4739e

13. Tsai TT, Nienaber CA, Eagle KA: Acute aortic syndromes. Circulation. 2005, 112:3802-3813. 10.1161/CIRCULATIONAHA. 105.534198

14. Marcaccio CL, Dumas RP, Huang Y, Yang W, Wang GJ, Holena DN: Delayed endovascular aortic repair is associated with reduced in-hospital mortality in patients with blunt thoracic aortic injury. J Vasc Surg. 2018, 68:64-73. 10.1016/j.jvs.2017.10.084

15. Dajee H, Richardson IW, Iype MO: Seat belt aorta: acute dissection and thrombosis of the abdominal aorta . Surgery. 1979, 85:263-267.

16. Shalhub S, Starnes BW, Tran NT, et al.: Blunt abdominal aortic injury. J Vasc Surg. 2012, 55:1277-1285. 10.1016/j.jvs.2011.10.132

17. Lock JS, Huffman AD, Johnson RC: Blunt trauma to the abdominal aorta. J Trauma. 1987, 27:674-677. 10.1097/00005373-198706000-00015

18. Glaser JD, Kalapatapu VR: Endovascular therapy of vascular trauma-current options and review of the literature. Vasc Endovascular Surg. 2019, 53:477-487. 10.1177/1538574419844073

19. Dayama A, Rivera A, Olorunfemi O, Mahmoud A, Fontecha CA, McNelis J: Open and endovascular abdominal aortic injury repair outcomes in polytrauma patients. Ann Vasc Surg. 2017, 42:156-161. 10.1016/j.avsg.2016.11.023

20. Cheaito A, Tillou A, Lewis C, Cryer H: Management of traumatic blunt IVC injury. Int J Surg Case Rep. 2016, 28:26-30. 10.1016/j.ijscr.2016.09.003

21. Buckman RF, Pathak AS, Badellino MM, Bradley KM: Injuries of the inferior vena cava . Surg Clin North Am. 2001, 81:1431-1447. 10.1016/s0039-6109(01)80016-5

22. Netto FA, Tien H, Hamilton P, et al.: Diagnosis and outcome of blunt caval injuries in the modern trauma center. J Trauma. 2006, 61:1053-1057. 10.1097/01.ta.0000241148.50832.87

23. Huerta S, Bui TD, Nguyen TH, Banimahd FN, Porral D, Dolich MO: Predictors of mortality and management of patients with traumatic inferior vena cava injuries. Am Surg. 2006, 72:290-296.

24. Hansen CJ, Bernadas C, West MA, Ney AL, Muehlstedt S, Cohen M, Rodriguez JL: Abdominal vena caval injuries: outcomes remain dismal. Surgery. 2000, 128:572-578. 10.1067/msy.2000.108054

25. Tsai R, Raptis C, Schuerer DJ, Mellnick VM: CT appearance of traumatic inferior vena cava injury . AJR Am J Roentgenol. 2016, 207:705-711. 10.2214/AJR.15.15870

26. Parke CE, Stanley RJ, Berlin AJ: Infrarenal vena caval injury following blunt trauma: CT findings . J Comput Assist Tomogr. 1993, 17:154 -157. 10.1097/00004728-199301000-00031

27. Rozycki GS, Kraut EJ: Isolated blunt rupture of the infrarenal inferior vena cava: the role of the ultrasound and computed tomography in an occult injury. J Trauma. 1995, 38:402-405. 10.1097/00005373-19950300000021

28. Matthees NG, Mankin JA, Kalinkin OM, Richardson RR: A rare opportunity for conservative treatment in a case of blunt trauma to the supradiaphragmatic inferior vena cava. J Surg Case Rep. 2013, 11:rjt092. 10.1093/jscr/rjt092

29. Bui TD, Mills JL: Control of inferior vena cava injury using percutaneous balloon catheter occlusion . Vasc Endovascular Surg. 2009, 43:490-493. 10.1177/1538574409339939

30. Castelli P, Caronno R, Piffaretti G, Tozzi M: Emergency endovascular repair for traumatic injury of the inferior vena cava. Eur J Cardiothorac Surg. 2005, 28:906-908. 10.1016/j.ejcts.2005.09.001

31. Watarida S, Nishi T, Furukawa A, et al.: Fenestrated stent-graft for traumatic juxtahepatic inferior vena cava injury. J Endovasc Ther. 2002, 9:134-7. 10.1177/152660280200900122 